\title{
EFECTOS DE DOS RECUBRIMIENTOS SOBRE LA CALIDAD DE LA PAPAYA (Carica papaya) VARIEDAD TAINUNG
}

\section{EFFECTS OF TWO COATINGS ON THE QUALITY OF PAPAYA (Carica papaya) VARIETY TAINUNG}

\author{
Abraham D. Miranda', Armando Alvis', Guillermo Arrazola² \\ Recibido para publicación: Julio 23 de 2013 - Aceptado para publicación: Marzo 13 de 2014

\section{RESUMEN}

Se comparó el efecto de la aplicación de recubrimientos, a base de almidón de yuca y uno comercial, en la conservación de la papaya (Carica papaya) variedad Tainung. El recubrimiento de almidón de yuca fue preparado a una concentración de $4 \% \mathrm{p} / \mathrm{v}$; la concentración de la cera comercial se preparó diluyendo una parte de cera con una parte de agua a temperatura ambiente. Las papayas se almacenaron acostadas (pedúnculo paralelo a la superficie), a temperatura ambiente $\left(22^{\circ} \mathrm{C} \pm 2^{\circ} \mathrm{C}\right)$ y humedad relativa del $85 \%$, por un periodo de 9 días. En el estudio del efecto del encerado en las papayas, solo hubo significancia estadística $(\mathrm{P}<0.05)$ sobre las variables: $\mathrm{pH}$ y firmeza; asimismo, la interacción entre los factores encerado y tiempo fue altamente significativo en las variables: $\mathrm{pH}$, ${ }^{\circ}$ Brix y firmeza. Además, en el efecto del tiempo sobre todas las variables observadas $\left(\mathrm{pH},{ }^{\circ}\right.$ Brix, acidez (\%), pérdida de masa y firmeza) existió diferencia estadísticamente significativa $(\mathrm{P}<0.05)$. Finalmente, se concluyó que en ambos recubrimientos y el control no se presentaron diferencias significativas en la acidez, sólidos solubles y pérdida de masa; sin embargo, estos fueron diferentes al control en el comportamiento de la firmeza y textura.

Palabras clave: Carica papaya, almidón de yuca, acidez

\begin{abstract}
The effect of the application of coatings based on cassava starch and one commercial in the conservation of papaya (Carica papaya) Tainung variety was compared. The coating cassava starch used was prepared with a concentration of $4 \% \mathrm{p} / \mathrm{v}$; the concentration of the commercial wax was prepared by diluting one part of wax to one part of water at room temperature according to the manufacturer's recommendations. Papayas are stored lying down (stalk parallel to the surface), at room temperature $\left(22^{\circ} \mathrm{C} \pm 2{ }^{\circ} \mathrm{C}\right)$ and $85 \%$ relative humidity for a period of 9 days. In the study of the effect of the wax in papayas, there was only statistically significant $(\mathrm{P}<0.05)$ on the variables: $\mathrm{pH}$ and firmness, also the interaction between waxed and time factors was highly significant in variables: $\mathrm{pH},{ }^{\circ} \mathrm{Brix}$ and firmness. Besides, in the effect of time on all observed variables $\left(\mathrm{pH},{ }^{\circ}\right.$ Brix, acidity $(\%)$, mass loss and firmness) there was a statistically significant difference $(\mathrm{P}<0.05)$. Finally, it was concluded that in both coatings and control did not differ significantly in acidity, soluble solids and mass loss, however these were different to the control on the behavior of the firmness and texture.
\end{abstract}

Key words: Carica papaya, cassava starch, acidity.

\footnotetext{
${ }^{1}$ Ingeniero de Alimentos, Facultad de Ingenierías, Universidad de Córdoba - Colombia.

${ }^{2}$ Grupo Investigación Procesos y Agroindustrias de Vegetales. Universidad de Córdoba. Colombia. Email: guillermo.arrazola@ua.es
} 


\section{INTRODUCCIÓN}

La papaya es una fruta perecedera que tiene una vida útil corta por presentar elevado contenido de humedad (alrededor del 90\%), textura susceptible a daños mecánicos y alta tasa respiratoria y de producción de calor (Sañudo et al. 2008). Esas características generan desventajas en cuanto a su manejo después de la cosecha, resultando en pérdidas derivadas de la falta de comercialización o de consumo del producto en tiempo hábil (Canto et al. 2006); según los comercializadores, las mayores pérdidas de la fruta son ocasionadas por mal manejo del fruto, resultando con magulladuras, maltrato, pudrición por antracnosis y otros hongos (15\%) y manipulación excesiva (75\%). Actualmente, los supermercados y almacenes de cadena tienen exigencias a la hora de comprar frutas y hortalizas; dentro de tales requerimientos está la aplicación de recubrimientos superficiales, especialmente ceras que reducen la tasa de respiración, la pérdida de agua y mejoran la apariencia, ya que dan brillo a la superficie (Elshiekh y AbuBakr 2008).

Los recubrimientos comestibles se definen (Navarros 2007) como productos comestibles que forman una fina capa sobre el alimento y se caracterizan por constituir una barrera semipermeable a los gases y al vapor de agua que retrasa el deterioro del alimento, mejoran las propiedades mecánicas, ayudan a mantener la integridad estructural del producto que envuelven, a retener compuestos volátiles y pueden actuar como vehículo de aditivos alimentarios (Tanada y Grosso 2005). Aplicados a las frutas permiten controlar la respiración y la senescencia de forma similar a las atmósferas modificadas, ejerciendo así una barrera a los gases y al vapor de agua. De esta manera se reduce el deterioro del fruto (Cisneros y Krochta 2002). Almidones de diferentes fuentes como trigo, maíz, papa y yuca se han utilizado, obteniendo, con este último, películas con buenas propiedades de flexibilidad y permeabilidad al vapor de agua (Parra et al. 2004). Canto (2006) evaluó el efecto del uso de almidón de yuca sobre la maduración de papaya a temperatura ambiente, vida útil, color superficial,actividad enzimática y encontró un retraso significativo en la maduración, pérdida de peso, cambios de color de la piel, firmeza, sólidos solubles y acidez titulable. Adicionalmente, se han elaborado recubrimientos incorporando glicerol aplicados sobre aguacate, y se ha encontrado un retraso en la maduración, mayor firmeza de la pulpa, retención del color de la piel y menor pérdida de peso (Aguilar 2005).

Teniendo en cuenta lo anterior, en esta investigación se desarrolló un recubrimiento a base de almidón para evaluar las características fisicoquímicas (acidez titulable (\%), sólidos solubles, $\mathrm{pH}$, pérdida de masa (\%) y firmeza) en papayas variedad Tainung cultivadas en el municipio de Valencia, departamento de Córdoba-Colombia.

Se comparó el efecto de la aplicación del recubrimiento a base de almidón de yuca con el producido por una cera comercial en la maduración de papaya (Carica papaya) variedad Tainung, con la finalidad de obtener un recubrimiento de bajo costo de elaboración, de fácil acceso a los pequeños y medianos productores de la región y que permita garantizar la calidad del producto. 


\section{MATERIALES Y MÉTODOS}

\section{Preparación de las muestras}

Se recolectaron papayas de los cultivos del municipio de Valencia, departamento de Córdoba-Colombia. Los frutos tenían una madurez fisiológica del 50\% (45\% del área superficial de la cascara amarilla) la cual se determinó visualmente basándose en una escala de color para estados de madurez de la papayas (Arias 2007). Además, los frutos fueron recolectados de plantas hermafroditas; estos frutos recogidos no presentaron ningún tipo de daño mecánico ni magulladuras, y fueron sometidos a un tratamiento de lavado con jabón para frutas a base de tenso-activos no iónicos, fórmula 1024 (Cerabrix) y luego sumergidos en una solución de cloro a $100 \mathrm{mg} \mathrm{L}^{-1}$ por 5 minutos y secados al aire (Canto et al. 2006).

\section{Preparación de los recubrimientos}

La concentración del recubrimiento de almidón de yuca fue del 4\% p/v (Amaya et al. 2010) modificado. Las soluciones se prepararon disolviendo 40 g de almidón de yuca en $960 \mathrm{~mL}$ de agua destilada, se adicionó 0,4 g de glicerol ( $1 \%$ base seca) y $0,12 \mathrm{~g}$ de polietilenglicol $(0,3 \mathrm{~g}$ de polietilenglicol por cada gramo de glicerol) el recubrimiento de almidón de yuca se preparó calentando la solución anterior, hasta que alcanzó $70^{\circ} \mathrm{C}$ con agitación constante y manteniendo esta temperatura hasta que alcanzó la gelificación (Amaya et al. 2010) que ocurrió entre 15 y 20 minutos. Luego se dejó enfriar hasta temperatura ambiente $\left(20^{\circ} \mathrm{C} \pm 2\right)$. La concentración de la cera comercial, que es una emulsión acuosa que se utilizó para tratamiento poscosecha, obtenida a partir de ceras vegetales, se preparó diluyendo una parte de cera con una parte de agua a temperatura ambiente, de acuerdo con las recomendaciones del fabricante.

\section{Aplicación de los recubrimientos}

La aplicación de la cera comercial (Cerabix) $®$ y el recubrimiento a base de almidón de yuca se realizó directamente sobre la superficie de la papaya Tainung con la ayuda de una brocha (Chiumarelli y Ferreira 2004). Posteriormente se secó por medio de aire forzado a temperatura ambiente (Restrepo 2009; Amaya et al. 2010).

\begin{abstract}
Almacenamiento
Las papayas con los recubrimientos y sin recubrimiento (control), se colocaron acostadas (pedúnculo paralelo a la superficie) sobre el mesón en una esponjilla de $1 \mathrm{~cm}$ de espesor previamente desinfectadas. Los frutos se almacenaron a temperatura y humedad relativa ambiente $\left(22^{\circ} \mathrm{C} \pm 2^{\circ} \mathrm{C}\right.$ y $\sim 85 \%$, respectivamente) por un periodo de 9 días.
\end{abstract}

\section{Análisis de las muestras}

El pH se midió por el método 981.12/90 de la A.O.A.C modificado; con un $\mathrm{pH}$ metro digital OAKTON, Ion 510 series. La acidez total se determinó por el método 942.05/90 de la A.O.A.C modificado. Los sólidos solubles totales fueron medidos según el método 932.12/90 de la A.O.A.C modificado, las lecturas se efectuaron con un refractómetro digital Pocket modelo B132363, rango 0-85\%; el cual expresa los resultados en grados Brix. La firmeza se midió con un penetrómetro digital BAREISS modelo HPE II, 63844 (5mm de diámetro). Los resultados se tomaron a partir del valor de la media de la fuerza aplicada a tres muestras de cada tratamiento, y expresados en la unidad de fuerza $\mathrm{cN}$ (centi Newton). La pérdida de masa se midió en una 
balanza digital OHAUS Adventurer (3100g \pm 0,1g) modelo ARC120, desde el día de inicio y se siguió registrando cada tres días hasta completar los nueve días. Los resultados se expresaron en porcentaje, calculados sobre el peso inicial, según la fórmula:

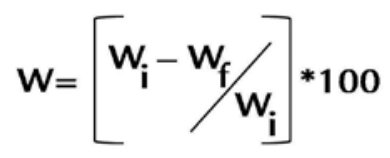

Dónde:

$\mathbf{W}$ es el porcentaje de pérdida de peso, $\mathbf{W}_{\mathbf{i}}$ es el peso inicial de la muestra y $\mathbf{W}_{\mathbf{f}}$ es el peso final de la muestra.

\section{Diseño experimental y análisis estadístico} Las muestras se distribuyeron con un arreglo factorial $3 * 4$ con 5 repeticiones (tres tratamientos: control, recubrimiento de almidón y cera comercial); (cuatro tiempos de almacenamiento: 0, 3, 6 y 9 días). El análisis de las muestras contó con 60 UE (unidades experimentales), considerando como UE una papaya con un recubrimiento determinado. Las variables independientes fueron el tipo de recubrimiento y tiempo de almacenamiento; las variables dependientes fueron: la pérdida de masa, firmeza, sólidos solubles ( $\left.{ }^{\circ} \mathrm{Brix}\right), \mathrm{pH}$ y porcentaje de acidez. Los resultados obtenidos fueron sometidos a análisis de varianza; para esto, se utilizó el Software estadístico SAS versión 9.1.3 y la prueba de comparación múltiple utilizada para separar medias de tratamientos fue Tukey con una probabilidad del 95\%.

\section{RESULTADOS Y DISCUSIÓN}

\section{Efecto de los tratamientos sobre el $\mathbf{p H}$}

La disminución del pH para las papayas del tratamiento control (desde 0 a 6 días) y de las papayas tratadas con almidón de yuca y cera comercial (desde los 3 a 9 días) durante el experimento (Tabla 1), se puede explicar con los resultados de Pinto et al. (2006) y Buitrago y Escobar (2009), los cuales establecen que la razón de la disminución del pH puede ser debido a la mayor actividad metabólica en el pico climatérico característico de la papaya, lo que llevaría a la síntesis de ácidos orgánicos de la fruta como el ácido abscísico, málico, cítrico, oxálico y otros; que se originan a partir de la ruptura de la pared celular (Castricini 2009).

El aumento no significativo del $\mathrm{pH}$ en el día 9 para las papayas del tratamiento control puede ser atribuido a una reducción de los ácidos presentes en la fruta, como corresponde a los resultados encontrados por Dayron et al. (2006). Este aumento es probablemente debido a la reducción de la actividad metabólica, la disminución de esta actividad es provocada por la menor difusión del oxígeno (Pinto et al. 2006); asimismo, ocurre con el incremento en la síntesis del contenido de aminoácidos (Katz et al. 2007). Castricini (2009), indica que el aumento del $\mathrm{pH}$ en las papayas luego de 8 días de almacenamiento está relacionado con el efecto tampón de compuestos, que se producen en respuesta a la mayor acidez que puede causar reacciones adversas a los frutos, tales como cambios en el sabor. El pH entre los días 0 y 3 presentado por los tratamientos con almidón de yuca pH $(5,49)$ y cera comercial $\mathrm{pH}(5,59)$ fueron mayores al control $\mathrm{pH}(5,3)$ y que se debe posiblemente a la alteración del proceso de respiración, en el cual es ralentizada la síntesis de ácidos orgánicos a partir de carbohidratos (Soler 2009). Esto 
se puede confirmar con el aumento en el contenido de sólidos solubles que se observa en el mismo periodo de tiempo de 0 a 3 días, debido a la degradación de polisacáridos en azúcares. Del mismo modo Téllez et al. (2007), obtuvieron en los estudios con curuba encerada con Primafresh (mezcla de ésteres ácido-grasos-sacarosa) un aumento en el pH. El factor encerado no provocó diferencias estadísticamente significativas en el $\mathrm{pH}$ para los tratamientos almidón de yuca y el control; sin embargo las papayas tratadas con la cera comercial si presentaron diferencias significativas con la prueba de Tukey $(\mathrm{P}<0.05)$ con respecto al $\mathrm{pH}$. Asimismo el factor tiempo de almacenamiento presentó solo diferencias significativas $(\mathrm{P}<0.05)$ a los 0 y 3 días con valores de $\mathrm{pH}$ de 5,38 y 5,49 respectivamente.

\section{Efectos de los tratamientos sobre la Acidez}

Los valores de acidez de las papayas en el tratamiento control no fueron significativos, pero tuvieron una tendencia a aumentar con los días de almacenamiento (Tabla 1), lo que concuerda con los resultados de Sañudo et al. (2008) y Amaya (2010). Este resultado no difiere de Costa y Balbino (2002) y Pinto et al. (2006) donde la acidez aumentó con los días de almacenamiento.

Esto se debe probablemente a la formación de ácido galacturónico, proveniente de la degradación de las pectinas, pues siendo el periodo de maduración de intensa actividad metabólica, los ácidos constituyen una excelente reserva energética del fruto a través de su oxidación en el ciclo de Krebs. Por lo tanto, la liberación de ácidos orgánicos de estas reacciones pueden aumentar la acidez (Castricini 2009).

Sin embargo, a partir del día 6 las papayas presentaron una estabilización de la acidez, que posiblemente se debe a la actividad de las deshidrogenasas y los ácidos orgánicos que son utilizados como sustratos de la respiración para la síntesis de nuevos componentes durante la maduración (Chitarra y Chitarra 2005; Botia et al. 2008; Sañudo et al. 2008; Álvarez et al. 2009).

Tabla 1.Resultados de $\mathrm{pH}$, acidez, ${ }^{\circ B}$ Brix, firmeza y pérdida masa de las papayas variedad Tainung.

\begin{tabular}{|c|c|c|c|c|c|c|}
\hline Tratamientos & Días & $\mathrm{pH}$ & Acidez (\%) & Brix & Firmeza $(\mathrm{cN})$ & Pérdida masa (g) \\
\hline \multirow{4}{*}{ Control } & 0 & $5,3 \pm 0,07$ & $0,065 \pm 0,002$ & $9,10 \pm 0,83$ & $75,76 \pm 4,81$ & 0,00 \\
\hline & 3 & $5,4 \pm 0,08$ & $0,064 \pm 0,003$ & $11,50 \pm 0,95$ & $50,45 \pm 3,09$ & $3,46 \pm 0,06$ \\
\hline & 6 & $5,5 \pm 0,07$ & $0,075 \pm 0,003$ & $11,66 \pm 0,98$ & $42,14 \pm 2,92$ & $7,45 \pm 0,09$ \\
\hline & 9 & $5,1 \pm 0,06$ & $0,084 \pm 0,003$ & $11,95 \pm 0,93$ & $24,55 \pm 1,80$ & $12,75 \pm 0,15$ \\
\hline \multirow{4}{*}{$\begin{array}{c}\text { Recubrimiento } \\
\text { con almidón }\end{array}$} & 0 & $5,3 \pm 0,07$ & $0,065 \pm 0,002$ & $9,10 \pm 0,83$ & $75,76 \pm 4,81$ & 0,00 \\
\hline & 3 & $5,5 \pm 0,06$ & $0,064 \pm 0,002$ & $11,50 \pm 0,94$ & $69,53 \pm 4,32$ & $1,68 \pm 0,04$ \\
\hline & 6 & $5,4 \pm 0,05$ & $0,070 \pm 0,002$ & $11,53 \pm 0,92$ & $44,87 \pm 2,98$ & $5,30 \pm 0,09$ \\
\hline & 9 & $5,3 \pm 0,04$ & $0,097 \pm 0,004$ & $11,58 \pm 0,93$ & $39,66 \pm 2,89$ & $9,54 \pm 0,10$ \\
\hline \multirow{4}{*}{$\begin{array}{c}\text { Recubrimiento } \\
\text { con cerabrix }\end{array}$} & 0 & $5,3 \pm 0,07$ & $0,065 \pm 0,002$ & $9,10 \pm 0,83$ & $75,76 \pm 4,81$ & 0,00 \\
\hline & 3 & $5,6 \pm 0,09$ & $0,064 \pm 0,002$ & $11,0 \pm 0,94$ & $64,51 \pm 4,78$ & $1,68 \pm 0,04$ \\
\hline & 6 & $5,5 \pm 0,06$ & $0,070 \pm 0,003$ & $11,36 \pm 0,95$ & $44,87 \pm 2,98$ & $1,87 \pm 0,05$ \\
\hline & 9 & $5,4 \pm 0,07$ & $0,097 \pm 0,004$ & $11,45 \pm 0,95$ & $42,87 \pm 2,93$ & $11,57 \pm 0,14$ \\
\hline
\end{tabular}


Por el contrario, las papayas con los tratamientos almidón de yuca y cera comercial inicialmente mantuvieron la acidez y posteriormente comenzaron a aumentar de forma significativa en el tiempo (Tabla 1). Según Amaya et al. (2010) puede ser debido a que los tratamientos retardaron o concentraron la volatilización de ácidos orgánicos, permitiendo que los frutos permanecieran más ácidos y más verdes. Pinto et al. (2006) señalan que la disminución de la acidez de la fruta se debe probablemente a la reducción de la actividad metabólica. En el último día de almacenamiento el aumento de la acidez puede estar relacionado con la mejora de la degradación de la pared celular debido a la actividad de la pectinametilesterasa (PME) (Castricini 2009).

Henrique y Evangelista (2006) observaron que después de cinco días de almacenamiento a $5^{\circ} \mathrm{C}$, en zanahorias mínimamente procesadas y cubiertas con película biodegradable, se produjo una disminución de la acidez en los primeros días y un aumento al final del almacenamiento.

\section{Efectos de los tratamientos sobre la firmeza}

La diferencia en el comportamiento de la firmeza se observa a través de la tendencia y los valores obtenidos de más de $70 \mathrm{cN}$, al medir el tratamiento con almidón (Figura 1) para las papayas de variedad Tainung en el tratamiento control, durante los 9 días. Estos resultados se pueden deber probablemente a que se produce mayor migración de vapor de agua a nivel de la superficie, favoreciendo el crecimiento de moho gris que genera daños estructurales en los tejidos permitiendo su ablandamiento. Asimismo, la pérdida de firmeza podría estar asociada a la degradación del parénquima cortical que conforma la pared celular debido al proceso de degradación enzimática (Pérez y Krochta 2000).

La actividad de las enzimas involucradas en la degradación de componentes de la pared celular se incrementa en muchas frutas, como en la papaya que es una fruta climatérica, donde el aumento en la respiración y la producción de etileno aumenta la actividad enzimática de la poligalacturonasa y la pectina provocando un efecto indirecto en la firmeza de la fruta (Castricini 2009).

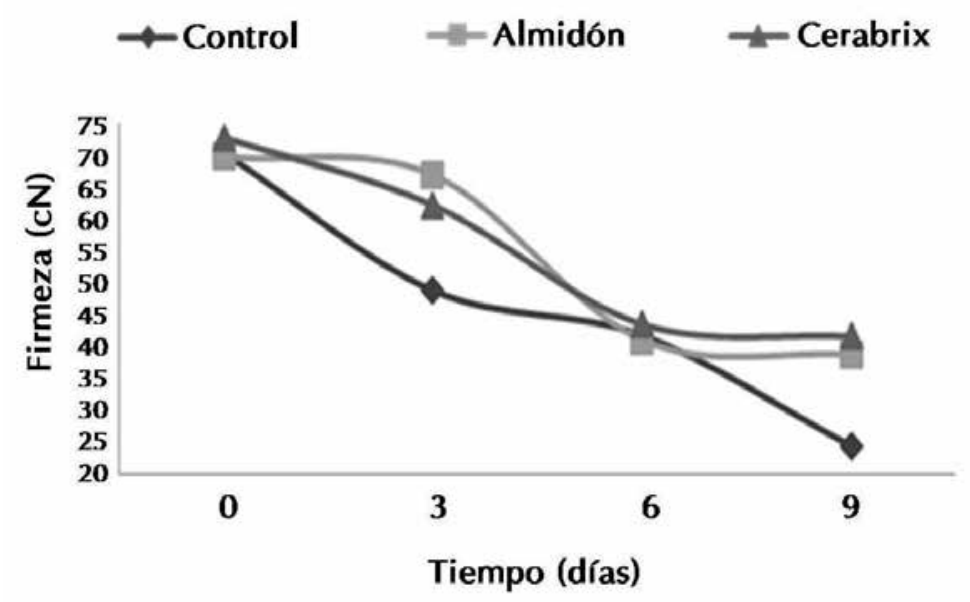

Figura 1. Comportamiento de la firmeza de papayas variedad Tainung tratadas con diferentes tipos de recubrimiento a través de 9 días de almacenamiento. 
La acción de las enzimas pectinolíticas que transforman la pectina soluble e insoluble promueve el ablandamiento del fruto (Márquez 2009).

Es de resaltar que el efecto de los tratamientos almidón de yuca y cera comercial, hayan provocado una disminución significativa en la perdida de firmeza de este fruto.

La firmeza de la pulpa a menudo está relacionada con la maduración, implicando que al progresar esta, la firmeza de la pulpa disminuye (Buitrago y Escobar 2009). Asimismo, Pinto et al. (2006) observaron una reducción de la pérdida de firmeza en papaya recubierta por la película de almidón al 1 y $3 \%$ durante el almacenamiento, que puede garantizar una mejor resistencia de la fruta a los daños mecánicos durante el manejo y en consecuencia, una mayor durabilidad. Castricini (2009) encontró que películas de almidón de yuca a altas concentraciones (3$5 \%$ ) pueden haber reducido el intercambio de gas en el fruto, por consiguiente la reducción de la frecuencia respiratoria y la producción de etileno, disminuyendo la pérdida de firmeza.

\section{Efectos de tratamientos en contenido de sólidos solubles totales (SST)}

En el contenido de sólidos solubles de las papayas recubiertas con almidón de yuca al $4 \% \mathrm{p} / \mathrm{v}$, se observa un incremento de los sólidos en la medida que transcurre el tiempo, inclusive para el control (Figura 2). Resultados que concuerdan con los de Castricini (2009), quien analizó el efecto del recubrimiento a base de almidón de yuca al 1, 3 y 5\% en papayas variedad Golden. No se conoce si la producción de sacarosa a partir del almidón es mínima, pero sí que contribuye al aumento marginal de los sólidos solubles después de la cosecha al degradarse a azúcares simples (Costa y Balbino 2002; Santamaria et al. 2009). Por otro lado, de acuerdo con Soler (2009), puede ocurrir un descenso en los sólidos solubles durante el almacenamiento, que se justifica por el consumo de carbohidratos en la síntesis de ácidos en la fruta.

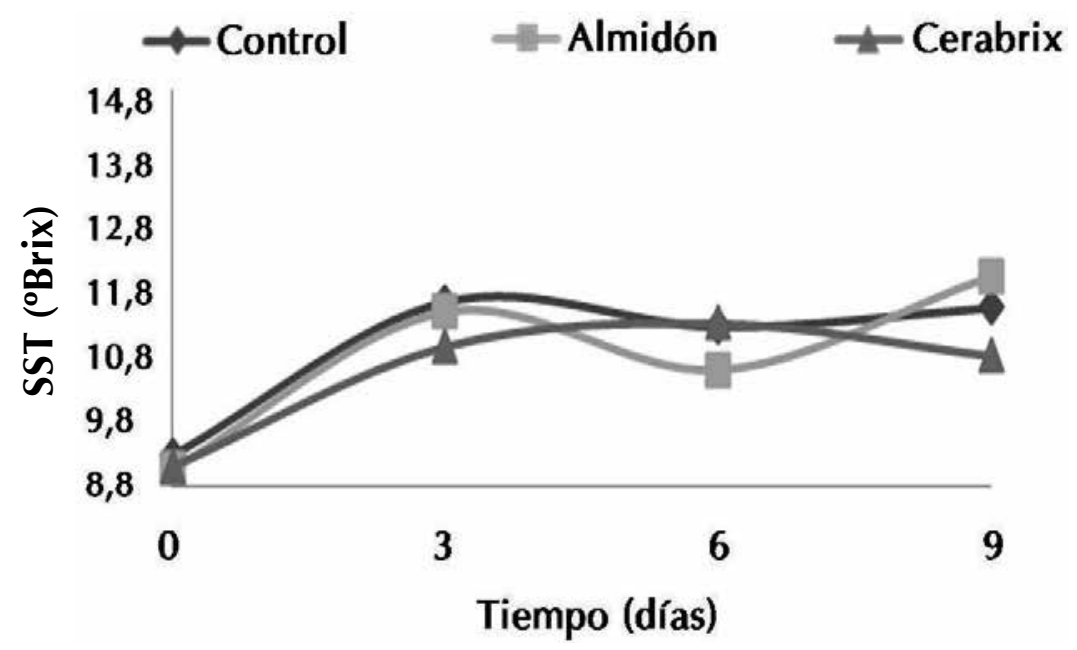

Figura 2. Comportamiento del contenido de SST ( ${ }^{\circ}$ Brix) de papayas variedad Tainung tratadas con diferentes tipos de recubrimiento durante 9 días de almacenamiento. 
En la figura 2 se puede observar como todas las 3 muestras alcanzaron en el noveno día de almacenamiento valores mayores a los indicados por Santamaria et al. (2009) de 10 ${ }^{\circ}$ Brix en papaya con madurez de consumo y un poco menor a los resultados por Sañudo et al. (2008) de 12,4 ${ }^{\circ}$ Brix. Resultados que concuerdan con Fonseca (2005), quien afirma que la variedad "Golden" en papaya, presenta sólidos solubles por encima de 12 en la etapa final de la maduración, lo que corresponde de 85 a 100\% de piel amarilla. Las papayas tratadas con almidón de yuca y cera comercial, mostraron cambios en el contenido de sólidos solubles inferiores a los mostrados por el tratamiento control, resultados similares son los encontrados por Téllez et al. (2007), los cuales obtuvieron que el efecto del encerado en curubas, presenta curvas con valores algo mayores cuando el fruto no está encerado, independientemente del cultivar, además de no encontrar diferencias significativas entre cultivares.

La apariencia totalmente amarilla de las papayas tratadas con cera comercial al sexto día de almacenamiento, puede deberse a un efecto más pronunciado de las papayas expuestas a la presencia del etileno exógeno que activó el metabolismo de conversión de almidón a azúcares (De la Cruz 2010), correspondiendo al punto máximo en los 'Brix alcanzados. Del mismo modo se puede decir que la cantidad de $11,9{ }^{\circ}$ Brix alcanzada a mitad del experimento, indica que el fruto climatérico sigue un proceso de maduración. El fruto comienza a incrementar su respiración y a utilizar sustratos como la glucosa en el proceso metabólico (Álvarez 2009). Asimismo ocurrió con las papayas del tratamiento control, las cuales presentaron en el día 3 la mayor concentración de SST, donde se pudo alcanzar posiblemente el aspecto de madurez de los frutos en los días 6 y 9.

\section{Efectos de los tratamientos en la pérdida de masa}

Los resultados obtenidos para la pérdida de masa de las papayas de variedad Tainung (Figura 3), concuerdan con las evidencias que encontró Buitrago y Escobar (2009); donde las concentraciones, de levaduras que aplicó en los frutos del banano cumplen con el mismo fin de retardar la maduración y el deshidratado a través del tiempo, aunque no fueron significativos los cambios. Asimismo, Dayron et al. (2006) encontró que los tratamientos con recubrimiento de almidón aplicados a las moras de castilla presentaron una disminución progresiva del porcentaje de pérdida de peso a lo largo de los días del almacenamiento. Del mismo modo durante el almacenamiento de frutos de curuba con o sin encerado, la tendencia general fue la pérdida de peso con el transcurso del tiempo (Téllez et al. 2007).

En los tratamientos en que se emplearon las películas de almidón de yuca y cera comercial, los porcentajes de pérdida de peso fueron menores, esta situación valida el efecto positivo de estos tipos de empaque primarios en la disminución del porcentaje de pérdida de peso de los frutos (Dayron 2006). Estos resultados también concuerdan con los encontrados por Petit-Jiménez (2010), quienes indican que el uso de cubiertas cerosas reduce la pérdida de peso. Cenci et al. (2002) han destacado que la pérdida de peso por encima del $5 \%$ es suficiente para el deterioro de las papayas. Esto se puede confirmar con lo observado en 
la figura 3 en la cual las papayas del control a partir del día 3 empiezan acercarse a 5\% de pérdida de peso, además el estado maduro presentado por las papayas desde el día 3 y con presencia de manchas en el día 9 reafirman la posibilidad del mencionado límite.

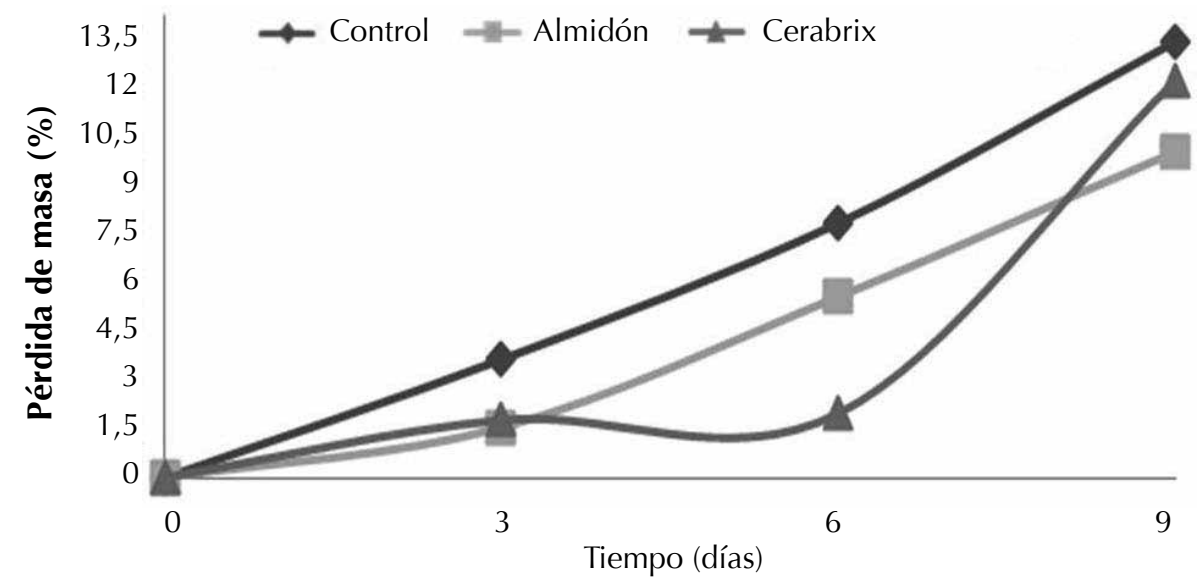

Figura 3. Comportamiento de la pérdida de masa (\%) de papayas variedad Tainung tratadas con diferentes tipos de recubrimientos a través de 9 días de almacenamiento.

\section{CONCLUSIONES}

Los efectos provocados por el recubrimiento elaborado a partir de almidón de yuca sobre las características fisicoquímicas (acidez y ${ }^{\circ}$ Brix) y pérdida de masa (\%) de las papayas variedad Tainung, no fueron estadísticamente diferentes a los provocados por el recubrimiento comercial (cerabrix $\left.{ }^{\circledR}\right)$ y el control a través de los 9 días de almacenamiento. Además los cambios provocados en la variación del comportamiento de la firmeza de las papayas recubiertas con la película de almidón de yuca elaborada, no presentaron cambios significativos a través del tiempo con respecto a la cera comercial (cerabrix), sin embargo estos son diferentes al control. La pérdida de masa al día 9 del control y los tratamientos fueron de 12,75; 9,54 y $11,57 \%$ respectivamente. El estado de la superficie de las papayas recubiertas con la película elaborada de almidón de yuca desde el noveno día de almacenamiento, presentó mejores características de firmeza, menor daño superficial comparado con los otros tratamientos (cerabrix y control). Los resultados demuestran que aplicaciones con la cera comercial son muy eficientes y pueden reducir la tasa respiratoria y mantener la textura y propiedades físicas de los frutos, incidiendo positivamente sobre el proceso de maduración al retardar su avance.

\section{REFERENCIAS}

Aguilar, M. 2005. Propiedades físicas y mecánicas de películas biodegradables y su empleo en el recubrimiento de frutos de aguacate. Centro de investigación en ciencia aplicada y tecnología avanzada. Instituto Politécnico Nacional. México D.F. 2005. p24-38.

Álvarez, J. Galvis, J. y Balaguera, H. 2009. Determinación de cambios físicos y químicos durante la maduración de 
frutos de champa (Campomanesia lineatilofia R. \& P.). Revista Agronomía Colombiana. 27(2): 253-259.

Amaya, P., Peña, L., Mosquera, A., Villada, H. y Villada, D. 2010. Efecto Del Uso De Recubrimientos Sobre La Calidad Del Tomate (Lycopersicon Esculentum Mill). Dyna.162: 67-73.

Arias, C. 2007. Manual de manejo postcosecha de frutas tropicales (Papaya, piña, plátano, cítricos). Organización de las Naciones Unidas Para la Agricultura y la Alimentación (FAO).

Botia, Y., Almanza, P. y Balaguera, H. 2008. Efecto de la temperatura sobre la maduración complementaria en curuba (Passiflora mollissima Bailey). Revista U.D.C.A Actualidad \& Divulgación Científica .11(2): 187-196.

Buitrago, J. y Escobar, A. 2009. Aplicación de Levadura Cándida spp. como una alternativa viable para la retardación en la pudrición del banano. Pontificia Universidad Javeriana, Bogotá D.C.

Canto, M., Santana, A., Da Rocha, A., Barbosa, D., Barbosa, S y dos Santos, V. 2006. Amadurecimento de Mamao Formosa Com Revestimento Comestível À Base De Fécula De Mandioca. Revista Ciênc. agrotec., Lavras. 6 (30): 1116-1119.

Castricini, A. 2009. Aplicação de Revestimentos Comestíveis para Conservação de Mamões (Carica papaya L.) 'Golden. Tesis Doctoral. Universidade Federal
Rural Do Rio De Janeiro, Instituto De Agronomia.

Cenci, S., Fonseca, M. y Freitas, O. 2002. Procedimentos póscolheita. In Mamão: pós-colheita. Revista Embrapa Informação Tecnológica. Série Frutas do Brasil 21(Capítulo 5): 24-38.

Chitarra, M. y Chitarra, A. 2005. Pós-colheita de frutas e hortaliças: fisiologia e manejo. Lavras, Brazil. 785 p.

Chiumarelli, M. y Ferreira, M. 2004. Utilização de ceras comestíveis para preservar a qualidade de tomates Débora armazenados em refrigeração. Memoria, XXXIII Congresso Brasileiro de Engenharia Agrícola.

Costa, A. y Balbino, J. 2002. Características da fruta para exportação e normas de qualidade. Embrapa Informação Tecnológica 12(1): 12-18.

Cisneros, Z., Krochta L. 2002. Internal modified atmospheres of coated fresh fruits and vegetables: understanding relative humidity effects. J. FoodSci. 67 (8): 2792-2797.

Dayron, A., Fischer, G. y Flórez, R. 2006. Almacenamiento refrigerado de frutos de mora de castilla (Rubus glaucus Benth.) en empaques con atmosfera modificada. Revista Agronomía Colombiana. 24 (2): 306-316.

De la Cruz, J., Vela, G., Dorantes, L. y García, H. 2010. Efecto del etileno sobre el ACC 
y ACC oxidasa en la maduración de la papaya maradol. Revista Fitotecnica Mexicana. 33(2): 133-140.

Elshiekh, F. y Abu-Bakr, A. 2008. Effect of Harvesting Method on Quality and Storability of Grapefruits., U. of K. J. Agric. Sci. 16(1): 1-14.

Fonseca, M. 2005. Escalas de maturação dos mamões "Sunrise Solo" e "Golden". In: XVII Congresso Brasileiro de Fruticultura. Viçosa, anais 2005.

Henrique, C. y Evangelista, M. 2006. Processamento mínimo de cenouras orgânicas com uso de películas biodegradáveis. Revista Ciências Exatas e da Terra, Ciências Agrárias e Engenharia. 12 (3): 7-14.

Katz, E., Fon, M., Lee, Y., Phinney, B., Sadka, A. y Blumwald, E. 2007. The citrus fruit proteome: insights into citrus fruit metabolism. Planta 226: 989-1005. http://www.pdfio.com./k588946.html\#

Márquez, C., Cartajena, J. y Pérez, M. 2009. Efecto De Recubrimientos Comestibles Sobre La Calidad En Poscosecha Del Níspero Japonés (Eriobotrya Japonica T.) VITAE, Revista de la facultad de química farmacéutica. 3 (16).

Navarros, T. 2007. Efecto de la composición de recubrimientos comestibles a base de hidroxiplimetilcelulosa y cera de abeja en la calidad de ciruelas, mandarinas y milojas. Universidad Politécnica de
Valencia. Departamento de tecnología de alimentos.

Parra, D., Tadini, C., Ponce, P. and Lugao, A. 2004. Mechanical properties and water vapor transmission in some blends of cassava starch edibles films. Carbohydrate Polymers. 58: 475-481.

Pérez, B. and Krochta J. 2000. Drying temperature effect on water vapor permeability and mechanical properties of whey proteinlipid emulsion films. Recista J. Agric Food Chem. (7): 2687-2692.

Petit, D., Terán, Y., Rojas, B., Salinas, R., García, J. y Báez, R. 2010. Efecto de las ceras comestibles sobre la calidad en frutos de papaya. Revista Iberoamericana de Tecnología Post-cosecha. 1 (11).

Pinto, A., Martins, M.,Dutra, E., vitorazi, I. y Pereira, S. 2006. Influência da atmosfera modificada por filmes plásticos sobre a qualidade do mamão armazenado sobre frigeração. Ciência e tecnologia de alimentos. 26(4): 744-748.

Restrepo, J. 2009. Conservación de fresa (fragaria $x$ ananassa duch cv. Camarosa) mediante la aplicación de recubrimientos comestibles de gel de mucilago de penca de sábila (Aloe Barbadensis Miller). Tesis de grado para optar el título de magíster en ciencia y tecnología de alimentos.

Santamaría, F., Díaz, R., Sauri, E., Espadas, F., Santamaría, J. y Larqué, A. 2009. Características de calidad de frutos de papaya Maradol en la madurez de 
consumo. Revista Agricultura Técnica en México. 35(3): 347-353.

\section{Sañudo, J., Siller, J., Osuna, T., Muy, D., Lopez,} G. y Labavitch, J. 2008. Control de maduración en frutos de papayas (Carica papaya L.) con 1-metilciclopropeno y 2-acido cloro etilfosfónico. Revista Fitotécnica Mexicana. 31(002): 141-147.

Soler, G. 2009. Cambios en la expresión genética asociados a la maduración interna del fruto de los cítricos: identificación de rutas metabólicas implicadas en acumulación y eliminación de ácidos. Tesis doctoral, Universidad Politécnica de Valencia, Valencia.
Tanada, S. and Grosso, C. 2005. Effect of edible wheat gluten-based films and coatings on refrigerated strawberry (Fragaria anassa quaility) Postharvest Biology and Technology. (36). 199-208.

Téllez, C., Fischer, G. y Quintero, $O$. 2007. Comportamiento fisiológico y fisicoquímico de frutos de curuba (Passiflora mollissima Bailey) encerados y almacenados a dos temperaturas. Revista Colombiana de Ciencias Hortícolas. 1(1): 67-80.

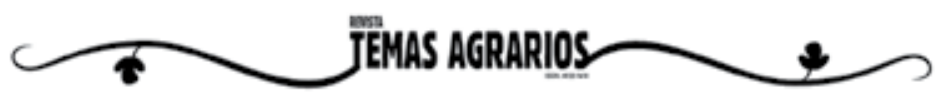

